\title{
Antigen-specific TIL therapy for melanoma: a flexible platform for personalized cancer immunotherapy
}

\author{
Sander Kelderman ${ }^{1 *}$, Bianca Heemskerk', Mireille Toebes ${ }^{1}$, Marit van Buuren', Nienke van Rooij ${ }^{1}$, Laura Bies $^{1}$, \\ Lorenzo Fanchi ${ }^{1}$, Lothar Germeroth², Pia Kvistborg ${ }^{1}$, Ton Schumacher ${ }^{1}$ \\ From Society for Immunotherapy of Cancer 28th Annual Meeting \\ National Harbor, MD, USA. 8-10 November 2013
}

Adoptive cell therapy (ACT) for melanoma has shown to be an effective treatment modality with clinical responses in approximately $50 \%$ of stage IV disease patients. However, durable complete responses are observed in only a small proportion (10-20\%) of patients. Recent data have demonstrated that the tumor-infiltrating lymphocyte (TIL) infusion products used for therapy harbor only low frequencies of $T$ cells reactive against presently known shared melanoma-associated epitopes. Furthermore, ongoing studies indicate that $\mathrm{T}$ cell reactivity against patient-specific mutated antigens may be common in human melanoma. Collectively, these two observations suggest that the development of a clinically applicable approach that allows the selection of defined tumor-reactive $T$ cell populations in a patient-specific manner would be of value. Here we develop such a platform technology and demonstrate its value in a preclinical model. First, reversible (strep-tagged) HLA-A*0201 molecules for clinical use were produced with a UV-sensitive peptide ligand, and the resulting MHC class I monomers could be loaded with peptides of interest in straightforward peptide exchange reactions. To subsequently evaluate the potential of these MHC exchange streptamers for antigen-specific $\mathrm{T}$ cell enrichment, we performed individual or combined enrichments of melanoma-reactive $\mathrm{T}$ cell populations from TIL populations. Enrichment of both high $(1 \geq \%)$ and low frequency $(<1 \%)$ tumor-specific $\mathrm{T}$ cell populations, either alone or combined, was shown to be feasible with a mean enrichment factor of 27 and 56 . To evaluate the potential of this technology for the generation of $\mathrm{T}$ cell products that recognize patient-specific neo-antigens, we performed combined enrichments of two HLA-A*0201 restricted T cell responses, directed against neo-antigens in the CDK4 (2.2\% of CD $8+$ cells $)$ and GCN1L1 (0.58\% of CD8+ cells) gene products that we identified by exome sequencing. Combined enrichment of these two patient-specific $\mathrm{T}$ cell populations resulted in a neo-antigen specific cell product with a purity of $92.4 \%$. Functional analysis of the neo-antigen selected $\mathrm{T}$ cell product against autologous tumor in NSG mice showed superior tumor control and survival compared to nonselected 'standard' TIL. Together, these data demonstrate that selection of (panels of) antigen-specific T cells using the combination of UV-induced ligand exchange and reversible streptamer technology is feasible. In combination with exome-driven analysis of tumor-specific $\mathrm{T}$ cell reactivity in human cancer, this strategy forms a highly flexible platform for the development of antigen-specific cell products for personalized cancer immunotherapy.

\section{Authors' details}

${ }^{1}$ Netherlands Cancer Institute NKI-AVL, Amsterdam, the Netherlands. ${ }^{2}$ Stage Cell Therapeutics, Göttingen, Germany.

Published: 7 November 2013

doi:10.1186/2051-1426-1-S1-P19

Cite this article as: Kelderman et al:: Antigen-specific TIL therapy for melanoma: a flexible platform for personalized cancer immunotherapy. Journal for ImmunoTherapy of Cancer 2013 1(Suppl 1):P19.

${ }^{1}$ Netherlands Cancer Institute NKI-AVL, Amsterdam, the Netherlands

Full list of author information is available at the end of the article 